\title{
Existence results for nonproper elliptic equations involving the Pucci operator
}

\author{
Alexander QUAAS ${ }^{1}$ and Boyan SIRAKOV
}

\begin{abstract}
We study the equation

$$
\left\{\begin{aligned}
-\mathcal{M}_{\lambda, \Lambda}^{+}\left(D^{2} u\right) & =f(x, u) & & \text { in } \Omega \\
u & =0 & & \text { on } \partial \Omega
\end{aligned}\right.
$$
\end{abstract}

in general smooth bounded domain $\Omega$ and show it possesses nontrivial solutions provided

- $f$ is sublinear, or

- $f$ is superlinear and the equation admits a priori bounds.

The existence result in the superlinear case is based on a new Liouville type theorem for $-\mathcal{M}_{\lambda, \Lambda}^{+}\left(D^{2} u\right)=u^{p}$ in a half-space.

MSC Numbers : 35J60, 35J65, 37C25

\section{Introduction}

This paper is a contribution to the study of uniformly elliptic fully nonlinear elliptic equations of the form

$$
F\left(x, u, D u, D^{2} u\right)=0
$$

The existence of solutions of (1.1) has been extensively investigated for coercive (or proper) uniformly elliptic operators $F$, mainly through adaptations of Perron's Method - see for example [12] and [11]. However, relatively little is known when the assumption of coercivity (that is, monotonicity in $u$ ) is dropped. On the other hand, when the second order operator is linear or in divergence form, a vast number of existence results are known.

In this paper, we focus on the model problem

$$
\left\{\begin{aligned}
-\mathcal{M}_{\lambda, \Lambda}^{+}\left(D^{2} u\right) & =f(x, u) & & \text { in } \quad \Omega, \\
u & =0 & & \text { on } \partial \Omega,
\end{aligned}\right.
$$

\footnotetext{
${ }^{1}$ Supported by FONDECYT, Grant N. 1040794, and ECOS grant C02E08.
} 
where $\Omega$ is a bounded regular domain in $\mathbb{R}^{N}$, and $\mathcal{M}_{\lambda, \Lambda}^{+}$is the extremal Pucci operator $([29])$, with parameters $0<\lambda \leq \Lambda$, defined by

$$
\mathcal{M}_{\lambda, \Lambda}^{+}(M)=\Lambda \sum_{e_{i}>0} e_{i}+\lambda \sum_{e_{i}<0} e_{i}
$$

for any symmetric $N \times N$ matrix $M$; here $e_{i}=e_{i}(M), i=1, \ldots, N$, denote the eigenvalues of $M$. All results we obtain can be restated for equation (1.2), with $\mathcal{M}_{\lambda, \Lambda}^{+}$replaced by $\mathcal{M}_{\lambda, \Lambda}^{-}\left(\mathcal{M}_{\lambda, \Lambda}^{-}\right.$is defined by exchanging the places of $\lambda$ and $\Lambda$ in (1.3)), see also the remark at the end of this section. Pucci's operators are extremal in the sense that

$$
\mathcal{M}_{\lambda, \Lambda}^{+}(M)=\sup _{A \in \mathcal{A}} \operatorname{tr}(A M), \mathcal{M}_{\lambda, \Lambda}^{-}(M)=\inf _{A \in \mathcal{A}} \operatorname{tr}(A M)
$$

where $\mathcal{A}$ denotes the set of all symmetric matrices whose eigenvalues lie in the interval $[\lambda, \Lambda]$. For more details on these operators we refer for example to the monograph of Cabré and Caffarelli [10]. Notice that $\mathcal{M}_{\lambda, \Lambda}^{+}$is not in divergence form.

Pucci's extremal operators appear for example in the context of stochastic control when the diffusion coefficient is a control variable, see for example the book of A. Bensoussan and J.L. Lions [4].

The study of (1.2) has been taken up only very recently in [18] and [31], where some results about existence of solutions in a ball or in a convex domain are proved (see Remark 2 after Theorem 1.6).

When $\lambda=\Lambda=1, \mathcal{M}_{\lambda, \Lambda}^{ \pm}$coincide with the Laplace operator, so that (1.2) becomes the classical equation

$$
\left\{\begin{aligned}
-\Delta u & =f(x, u) & & \text { in } \Omega, \\
u & =0 & & \text { on } \partial \Omega .
\end{aligned}\right.
$$

For this equation and, in general, for equations involving divergence form operators existence results can be obtained by variational methods - see for example the survey papers [25], [30]. Another successful approach for studying existence of solutions of (1.5) are topological methods. General references on this topic are the book [15] and the survey paper [27].

Our approach to study the existence problem for (1.2) falls into the group of topological methods and is based on the degree theory for compact operators in positive cones (Kransnoselskii's index). This approach has been successfully applied by many authors to a variety of problems. Of special interest to us is the work of de Figueiredo, Lions and Nussbaum [19], where appears an abstract existence theorem on which we base our arguments (see Theorem 4.1 in Section 4). 
Next we list our results. A standing assumption on the nonlinearity $f(x, u)$ will be the following condition.

$\left(\mathbf{f}_{\mathbf{0}}\right) f$ is a Hölder continuous function on $\bar{\Omega} \times[0, \infty)$, such that $f(x, 0)=0$ and $f(x, s) \geq-\gamma s$ for some $\gamma \geq 0$ and all $s \geq 0, x \in \bar{\Omega}$.

First, we show that (1.2) has a positive solution provided the problem is sublinear, in the sense that

$\left(\mathbf{H}_{\mathbf{0}}\right) \quad \limsup _{u \rightarrow \infty} \frac{f(x, u)}{u}<\mu_{1}^{+}<\liminf _{u \rightarrow 0} \frac{f(x, u)}{u} \leq \infty, \quad$ uniformly in $x \in \bar{\Omega}$.

Here $\mu_{1}^{+}>0$ denotes the first eigenvalue of the Pucci operator $\mathcal{M}_{\lambda, \Lambda}^{+}$, associated to a positive eigenfunction. The existence of $\mu_{1}^{+}$is studied by Felmer and Quaas in [18] when $\Omega$ is ball, and by Quaas in [31] for any regular bounded domain. More properties of $\mu_{1}^{+}$are estabished in the recent paper by Busca, Esteban and Quaas [7]. In Section 2 we quote the results from these papers that we need.

Theorem 1.1 Suppose $\left(f_{0}\right)$ and $\left(H_{0}\right)$ hold. Then problem (1.2) has a positive classical solution.

Remark 1. A typical nonlinearity which satisfies $\left(f_{0}\right)$ and $\left(H_{0}\right)$ is the function $f(x, u)=a(x) u^{p}$, where $0<p<1$ and $a(x)$ is bounded between two positive constants.

Remark 2. Theorem (1.1) seems to be the first result in the literature which concerns sublinear equations involving the Pucci operator.

Next we turn to superlinear equations, that is, equations in which the nonlinearity satisfies

$\left(\mathbf{H}^{\mathbf{0}}\right) \quad \limsup _{u \rightarrow 0} \frac{f(x, u)}{u}<\mu_{1}^{+}<\liminf _{u \rightarrow \infty} \frac{f(x, u)}{u} \leq \infty, \quad$ uniformly in $x \in \bar{\Omega}$.

In order to state the existence theorem, we consider the family of problems obtained from $(1.2)$ by replacing $f(x, u)$ with $f(x, u+t)$, for $t \geq 0$. Let $A_{t}$ denote the set of nonnegative classical solutions for any such problem and let $\mathcal{S}_{t}=\underset{0 \leq s \leq t}{\cup} A_{s}$.

Theorem 1.2 Suppose $\left(f_{0}\right)$ and $\left(H^{0}\right)$ hold. Suppose in addition that for each $t \geq 0$ there exist a constant $C$ depending only on $t, \Omega$ and $f$ such that

$$
\|u\|_{L^{\infty}(\Omega)} \leq C \quad \text { for all } \quad u \in \mathcal{S}_{t} .
$$

Then problem (1.2) has a positive classical solution. 
Remark. In the sequel we shall consider nonlinearities with power-like growth at infinity, in which case it is enough to have condition (1.6) only for $t=0$, that is, to assume equation (1.2) admits a priori bounds.

Theorem 1.2 settles the existence question provided a priori bounds exist. Consequently, we next concentrate on getting such bounds for (1.2). We use the blow-up method of Gidas and Spruck, which has turned to be the most powerful tool for obtaining a priori bounds in more classical situations. The most important drawback of this method is that it depends on availability of non-existence results (we shall refer to these as Liouville type theorems) for equations of type (1.2), when $\Omega$ is the whole space or a half-space, and such results are often difficult to get. We note that ever since the fundamental work of Gidas and Spruck [21] there has been a multitude of Liouville type results for equations of the type $-\Delta u+f(u)=0$.

Let us recall the recent progress in proving Liouville type theorems for equations involving Pucci's operator, a very interesting question by itself.

First, Cutri and Leoni [13] studied the problem

$$
\begin{aligned}
\mathcal{M}_{\lambda, \Lambda}^{+}\left(D^{2} u\right)+u^{p} & =0 \text { in } \mathbb{R}^{N}, \\
u & \geq 0 \text { in } \mathbb{R}^{N},
\end{aligned}
$$

where $p>1$. They obtained the following Liouville type theorem.

Theorem 1.3 (Cutri-Leoni) Suppose $N \geq 3$ and set

$$
p^{+}:=\frac{\tilde{N}}{\tilde{N}-2}, \quad \text { with } \quad \tilde{N}=\frac{\Lambda}{\lambda}(N-1)+1 .
$$

If $1<p \leq p^{+}$then the only viscosity supersolution of (1.7) is $u \equiv 0$.

Next, in the radial case a Liouville type theorem for a larger range of $p$ can be obtained for solutions (as opposed to just supersolutions) of (1.7). Felmer and Quaas proved the following theorem in [17].

Theorem 1.4 (Felmer-Quaas) Let $N \geq 3$. Then there exist a number $p_{*}^{+}>p^{+}>1$ such that if $1<p<p_{*}^{+}$then (1.7) does not have a non trivial radially symmetric classical solution.

When the parameters $\lambda$ and $\Lambda$ are equal, one gets $p_{*}^{+}=p_{N}$, where $p_{N}=$ $(N+2) /(N-2)$ is the usual Sobolev critical exponent. Note that in the case $\lambda<\Lambda$ we have $p_{*}^{+}>\max \left\{p_{N}, p^{+}\right\}$, so there is a gap between the exponents of Theorem 1.3 and Theorem 1.4. It is an open problem to show that (1.7) has no solutions in the range $p^{+}<p<p_{*}^{+}$(the result of Gidas and Spruck states that this is the case when $\lambda=\Lambda$ ). 
Another important type of nonexistence results concern problems in a half space. In particular, they are needed for the blow-up method to work in arbitrary smooth domains. This question has been completely open up to now for fully nonlinear equations.

We establish the following Liouville type theorem in the half space. We denote $\mathbb{R}_{+}^{N}=\left\{x \in \mathbb{R}^{N} \mid x_{N}>0\right\}$.

Theorem 1.5 Suppose $N \geq 3$ and set

$$
\tilde{p}^{+}=\frac{\Lambda(N-2)+\lambda}{\Lambda(N-2)-\lambda} .
$$

Then the problem

$$
\begin{aligned}
\mathcal{M}_{\lambda, \Lambda}^{+}\left(D^{2} u\right)+u^{p} & =0 \quad \text { in } \mathbb{R}_{+}^{N} \\
u & =0 \text { on } \partial \mathbb{R}_{+}^{N} .
\end{aligned}
$$

does not have a nontrivial nonnegative bounded solution, provided $1<p \leq$ $\tilde{p}^{+}$. Observe that $\tilde{p}^{+}>p^{+}$, so Theorem 1.5 is valid for a larger range of $p$ in comparison with Theorem 1.3.

A theorem of this type for the equation $-\Delta u+f(u)=0$ was first obtained by Dancer in [14]. We are going to prove Theorem 1.5 by using a (simplified) version of the proof of Berestycki, Caffarelli and Nirenberg [6], who showed that solutions of $-\Delta u+f(u)=0$ in a half space which are at most exponential at infinity are necessarily monotone in $x_{N}$. Once this is proved we show that it is possible to pass to the limit as $x_{N} \rightarrow \infty$, and that this leads us to a solution of the same problem in $\mathbb{R}^{N-1}$, which permits the use of Liouville type theorems in the whole space.

Remark. The monotonicity results used in proof of Theorem 1.5 can be applied to much more general nonlinearities. See Theorem 3.1 in Section 3.

The following existence result is a consequence of Theorem 1.2 and the Liouville type results.

Theorem 1.6 Assume $N \geq 3, f$ does not depend on $x$, satisfies the hypotheses $\left(f_{0}\right),\left(H^{0}\right)$, and

$\left(\mathbf{f}_{\mathbf{1}}\right)$ there exist $p \in\left(1, p^{+}\right]$and a constant $C^{*}>0$ such that

$$
\lim _{s \rightarrow+\infty} \frac{f(s)}{s^{p}}=C^{*}
$$

Then there exists a positive classical solution of (1.2). 
Remark 1. The range of $p$ in $\left(f_{1}\right)$ is given by the range of nonexistence of solutions of (1.7). Should this range be subsequently extended, our results would automatically imply that Theorem 1.6 holds for $p$ in the new range.

Remark 2. Using an argument based on Theorem 1.4 Felmer and Quaas showed that Theorem 1.6 is valid for $p \in\left(1, p_{*}^{+}\right)$, provided $\Omega$ is a ball, see [17] and [18]. In this case the problem admits a radial positive solution. Further, Theorem 1.4 was proved in [31] in the case of a convex domain. In this case, the maximum of the solution to (1.2) is away from the boundary so the Liouville type theorem in the half space is not needed to establish the a priori bounds.

The plan of the paper is as follows. In Section 2 we review some known results about linear operators and Pucci's operator. In Section 3 we prove a monotonicity result and the Liouville type theorem in the half space, Theorem 1.5. In Section 4 we describe the abstract setting that we use, and deduce our existence results.

We stress once more that all results can be restated for $\mathcal{M}_{\lambda, \Lambda}^{-}$instead of $\mathcal{M}_{\lambda, \Lambda}^{+}$, replacing the first eigenvalue $\mu_{1}^{+}$and the exponents $p^{+}$by the corresponding values for $\mathcal{M}_{\lambda, \Lambda}^{-}$. Finally, our results extend to more general equations of the type $F\left(D^{2} u, D u\right)=f(x, u)$, provided one can define a suitable notion of first eigenvalue for the uniformly elliptic operator $F$.

\section{Preliminaries}

We start by recalling a classical lemma of C. Pucci, see [29] or [10].

Lemma 2.1 For a fixed function $v \in W_{l o c}^{2, N}(\Omega)$ there exists a symmetric measurable matrix $A(x) \in \mathcal{A}(\mathcal{A}$ is defined in (1.4)), such that

$$
\mathcal{M}_{\lambda, \Lambda}^{+}\left(D^{2} v\right)=L_{A} v
$$

where $L_{A}$ is the second order linear elliptic operator associated to $A$, that is $L_{A}=\sum a_{i j}(x) \partial_{i j}=\operatorname{tr}\left(A D^{2}(\cdot)\right)$. The same result holds for $\mathcal{M}_{\lambda, \Lambda}^{-}$.

Let $L=L_{A}+\sum b_{i} \partial_{i}+c(x)$ be a linear elliptic operator in nondivergence form with bounded measurable coefficients. We are going to use the following Harnack-type inequality, obtained by Krylov and Safonov, see for example [24]. 
Theorem 2.1 Let $B_{R}$ be a ball of radius $R$ in $\mathbb{R}^{N}$, and denote by $B_{2 R}$ the concentric ball of radius $2 R$. Let $u \in W^{2, N}\left(B_{2 R}\right)$ and $f \in L^{N}\left(B_{2 R}\right)$ satisfy $u \geq 0$ in $B_{2 R}$ and $L u=f$ in $B_{2 R}$. Then

$$
\sup _{B_{R}} u \leq C\left\{\inf _{B_{R}} u+R\|f\|_{L^{N}\left(B_{2 R}\right)}\right\},
$$

where $C$ depends only on $N, \Lambda, \lambda$, and on the bounds for $c(x)$ and $b_{i}(x)$.

Now we state a consequence of a maximum principle for narrow unbounded domains, obtained by Cabré (see [8] and Theorem 5.3 in [9]).

Theorem 2.2 Suppose that $\Omega$ is between two parallel hyperplanes at a distance $d$. Then the maximum principle holds for $L=L_{A}+\sum b_{i} \partial_{i}+c(x)$ in $\Omega$, provided that $d$ is small enough (depending only on bounds for the coefficients of $L$ ).

The following version of Hopf's boundary lemma holds.

Lemma 2.2 Let $\Omega$ be a regular domain and let $u \in W_{\text {loc }}^{2, N}(\Omega) \cap C(\bar{\Omega})$ be a non-negative solution to

$$
\mathcal{M}_{\lambda, \Lambda}^{-}\left(D^{2} u\right)+c(x) u \leq 0 \quad \text { in } \quad \Omega
$$

with $c(x) \in L^{\infty}(\Omega)$. Then either $u$ vanishes identically in $\Omega$ or $u(x)>0$ for all $x \in \Omega$. Moreover, in the latter case for any $x_{0} \in \partial \Omega$ such that $u\left(x_{0}\right)=0$,

$$
\limsup _{t \searrow 0} \frac{u\left(x_{0}-t \nu\right)-u\left(x_{0}\right)}{t}<0,
$$

where $\nu$ is the outer normal to $\partial \Omega$.

Remark. For a general strong maximum principle for degenerate convex elliptic operators, see the paper of M. Bardi, F. Da Lio [2].

We are going to use the following results for Pucci operators (for the proofs of which we refer for example to [10] and [11]).

Theorem 2.3 If the function $u$ is a viscosity solution to the equation

$$
-\mathcal{M}_{\lambda, \Lambda}^{+}\left(D^{2} u\right)=g(x)
$$

in a ball $B_{2 R}$ and $g \in L^{p}\left(B_{2 R}\right)$ for some $p \geq N$ then $u \in W^{2, p}\left(B_{R}\right)$ and one has the interior estimate

$$
\|u\|_{W^{2, p}\left(B_{R}\right)} \leq C\left(\|u\|_{L^{\infty}\left(B_{2 R}\right)}+\|g\|_{L^{p}\left(B_{2 R}\right)}\right) .
$$


If $g \in C^{\alpha}$ for some $\alpha \in(0,1)$ then $u \in C^{2, \alpha}$ and

$$
\|u\|_{C^{2, \alpha}\left(B_{R}\right)} \leq C\left(\|u\|_{L^{\infty}\left(B_{2 R}\right)}+\|g\|_{C^{\alpha}\left(B_{2 R}\right)}\right) .
$$

In addition, if (2.10) is satisfied in a regular domain and $u=0$ on the boundary of the domain then $u$ satisfies a $C^{\alpha}$-estimate up to the boundary.

We note that the results in Theorem 2.3 strongly depend on the fact that Pucci's operator is a convex function of the Hessian.

Theorem 2.4 Suppose $u_{n}$ and $g_{n}$ are sequences of continuous functions such that $u_{n}$ is a solution (or subsolution, or supersolution) of the equation

$$
-\mathcal{M}_{\lambda, \Lambda}^{+}\left(D^{2} u_{n}\right)=g_{n}(x)
$$

in a domain $\Omega$. Suppose $u_{n}$ and $g_{n}$ converge uniformly on compact subsets of $\Omega$ to functions $u$ and $g$. Then $u$ is a solution (or subsolution, or supersolution) in $\Omega$ of

$$
-\mathcal{M}_{\lambda, \Lambda}^{+}\left(D^{2} u\right)=g(x)
$$

Recently, the following results on existence of first eigenvalue for the Pucci operator were proven in [31] and [7].

Theorem 2.5 Consider the problem

$$
\left\{\begin{aligned}
\mathcal{M}_{\lambda, \Lambda}^{+}\left(D^{2} u\right)+\mu u & =0 \text { in } \Omega \\
u & >0 \text { in } \Omega . \\
u & =0 \text { on } \partial \Omega .
\end{aligned}\right.
$$

If we define

$$
\begin{aligned}
\mu_{1}^{+}= & \sup _{\phi}\left\{\mu \in \mathbb{R} \mid \exists \phi \in W_{\text {loc }}^{2, N}(\Omega) \cap C(\bar{\Omega}) \quad\right. \text { such that } \\
& \left.\phi>0 \text { and } \mathcal{M}_{\lambda, \Lambda}^{+}\left(D^{2} \phi\right)+\mu \phi \leq 0 \quad \text { in } \Omega\right\}
\end{aligned}
$$

then there exists a function $\varphi_{1}^{+} \in C^{2}(\Omega) \cap C(\bar{\Omega})$ such that the couple $\left(\mu_{1}^{+}, \varphi_{1}^{+}\right)$ is a solution of (2.11). In addition, any other couple $(\mu, u)$ which satisfies (2.11) is of the form $\left(\mu_{1}^{+}, k \varphi_{1}^{+}\right)$, for some $k>0$.

Theorem 2.6 The operator $\mathcal{M}_{\lambda, \Lambda}^{+}\left(D^{2} \cdot\right)+\mu$ satisfies the maximum principle for $\mu<\mu_{1}^{+}$, in the sense that if $u \in W_{\text {loc }}^{2, N}(\Omega) \cap C(\bar{\Omega})$ is a solution of

$$
\left\{\begin{aligned}
\mathcal{M}_{\lambda, \Lambda}^{+}\left(D^{2} u\right)+\mu u & \geq 0 \text { in } \Omega \\
u & \leq 0 \text { on } \partial \Omega
\end{aligned}\right.
$$

for some $\mu<\mu_{1}^{+}$, then $u \leq 0$ in $\Omega$. 


\section{A Liouville type theorem in the half space}

Theorem 3.1 Suppose we have a non trivial classical bounded solution of

$$
\left\{\begin{aligned}
\mathcal{M}_{\lambda, \Lambda}^{+}\left(D^{2} u\right)+f(u) & =0 \text { in } \mathbb{R}_{+}^{N} \\
u & \geq 0 \text { in } \mathbb{R}_{+}^{N} \\
u & =0 \text { on } \partial \mathbb{R}_{+}^{N},
\end{aligned}\right.
$$

where $f(u)$ is a locally Lipschitz continuous function with $f(0) \geq 0$. Then

$$
\frac{\partial u}{\partial x_{N}}>0 \text { in } \mathbb{R}_{+}^{N}
$$

Proof. Suppose $u$ is a solution of (3.13), $u \neq \equiv 0,0 \leq u \leq M$. Note that $u$ satisfies the equation

$$
\mathcal{M}_{\lambda, \Lambda}^{+}\left(D^{2} u\right)+c(x) u=-f(0) \leq 0 \quad \text { in } \quad \Omega,
$$

where $c(x) \in L^{\infty}(\Omega)(c(x)$ is bounded by a Lipschitz constant of $f$ on $[0, M])$ is defined by

$$
c(x)=\frac{f(u(x))-f(0)}{u(x)} \quad \text { if } u(x) \neq 0, \quad c(x)=0 \quad \text { if } u(x)=0 .
$$

Hence $u$ is strictly positive in $\mathbb{R}_{+}^{N}$, by Lemma 2.2 .

We use the moving planes method of Alexandrov [1], developed in the framework of partial differential equations by Serrin [32], Gidas-Ni-Nirenberg [20], and Berestycki-Nirenberg [5].

For each $\beta$ we denote

$$
T_{\beta}=\left\{x \in \mathbb{R}_{+}^{N} \quad \mid \quad x_{N}=\beta\right\}, \quad \Sigma_{\beta}=\left\{x \in \mathbb{R}_{+}^{N} \quad \mid \quad 0<x_{N}<\beta\right\}
$$

and introduce the functions

$$
u_{\beta}(x)=u\left(y, 2 \beta-x_{N}\right), \quad w_{\beta}(x)=u_{\beta}(x)-u(x), \quad x=\left(y, x_{N}\right),
$$

defined in $\Sigma_{\beta}$. Since for any pair of symmetric $N \times N$ matrices $M, N$ the inequality

$$
\mathcal{M}_{\lambda, \Lambda}^{-}(M-N) \leq \mathcal{M}_{\lambda, \Lambda}^{+}(M)-\mathcal{M}_{\lambda, \Lambda}^{+}(N)
$$

holds, we have that $w_{\beta}$ satisfies

$$
\mathcal{M}_{\lambda, \Lambda}^{-}\left(D^{2}\left(w_{\beta}\right)\right)+c_{\beta}(x) w_{\beta} \leq 0 \quad \text { in } \quad \Sigma_{\beta},
$$


where $c_{\beta}(x) \in L^{\infty}(\Omega)$ is defined by

$$
c_{\beta}(x)=\frac{f\left(u_{\beta}(x)\right)-f(u(x))}{u_{\beta}(x)-u(x)} \quad \text { if } u_{\beta}(x) \neq u(x)
$$

and $c(x)=0$ if $u_{\beta}(x)=u(x)$. We clearly have $w_{\beta} \geq 0$ on $\partial \Sigma_{\beta}$. By Lemma 2.1 we can use Theorem 2.2 to infer that if $\beta$ is small enough then $w_{\beta} \geq 0$ in $\Sigma_{\beta}$. Hence

$$
\beta^{*}=\sup \left\{\beta \mid w^{\mu} \geq 0 \quad \text { in } \quad \Sigma_{\mu} \quad \forall \mu<\beta\right\}>0 .
$$

Using Hopf's lemma we conclude that $w_{\beta}>0$ in $\Sigma_{\beta}$ and

$$
\frac{\partial u}{\partial x_{N}}=-\frac{1}{2} \frac{\partial w_{\beta}}{\partial x_{N}}>0 \quad \text { on } \quad T_{\beta}
$$

for each $0<\beta \leq \beta^{*}$. Therefore, the theorem is proved if we show that $\beta^{*}=+\infty$.

Suppose for contradiction that $\beta^{*}$ is finite. By Lemma 2.1 and Theorem 2.2 we can fix $\varepsilon_{0}>0$ such that $\mathcal{M}_{\lambda, \Lambda}^{-}\left(D^{2} \cdot\right)+c_{\beta}(x)$ satisfies the maximum principle in the domain $\Sigma_{\beta^{*}+\varepsilon_{0}} \backslash \Sigma_{\beta^{*}-\varepsilon_{0}}$.

Lemma 3.1 There exist $\delta_{0} \in\left(0, \varepsilon_{0}\right]$, such that for each $\delta \in\left(0, \delta_{0}\right)$ we have

$$
w_{\beta^{*}+\delta} \geq 0 \quad \text { in } \quad \Sigma_{\beta^{*}-\varepsilon_{0}} \backslash \Sigma_{\varepsilon_{0}}
$$

Suppose this lemma is proved. Then we can apply Theorem 2.2 and lemma 2.1 to inequality (3.15) in $\Sigma_{\beta^{*}+\delta} \backslash \Sigma_{\beta^{*}-\varepsilon_{0}}$ and in $\Sigma_{\varepsilon_{0}}$ to conclude that $w_{\beta^{*}+\delta} \geq 0$ in $\Sigma_{\beta^{*}+\delta}$ for each $\delta \in\left(0, \delta_{0}\right)$. This contradicts the maximal choice of $\beta^{*}$ and proves Theorem 3.1 .

Proof of Lemma 3.1. Suppose the Lemma is false, that is, there exist sequences $\delta_{m} \rightarrow 0$ and $x^{(m)}=\left(y^{(m)}, x_{N}^{(m)}\right) \in \Sigma_{\beta^{*}-\varepsilon_{0}} \backslash \Sigma_{\varepsilon_{0}}$ such that

$$
w_{\beta^{*}+\delta_{m}}\left(x^{(m)}\right)<0 \text {. }
$$

We can suppose that $x_{N}^{(m)} \rightarrow x_{N}^{0} \in\left[\varepsilon_{0}, \beta^{*}-\varepsilon_{0}\right]$ as $m \rightarrow \infty$.

We define the functions

$$
u^{(m)}\left(y, x_{N}\right)=u\left(y+y^{(m)}, x_{N}\right)
$$

and, respectively

$$
w_{\beta}^{(m)}\left(y, x_{N}\right)=u^{(m)}\left(y, 2 \beta-x_{N}\right)-u^{(m)}\left(y, x_{N}\right) .
$$


Note that $u^{(m)}$ satisfies the same equation as $u$, and respectively an equation like (3.14). So we can infer from Theorem 2.3 that

$$
\left\|u^{(m)}\right\|_{W^{2, p}(K)} \leq C,
$$

for each compact set $K$ in the closure of $\mathbb{R}_{+}^{N}$ (the constant $C$ depends on $K$, $M$, and on a Lipschitz constant of $f$ on $[0, M])$. It follows from embedding theorems, together with Theorems 2.3 and 2.4 , that $u^{(m)}$ converges uniformly to a classical solution $\tilde{u}$ of (3.13), and $\tilde{u}$ also satisfies an equation like (3.14).

By the strong maximum principle we have that either $\tilde{u}$ is strictly positive in $\mathbb{R}_{+}^{N}$ (note that this is the only possibility if $f(0) \neq 0$ ) or $\tilde{u}$ vanishes identically in $\mathbb{R}_{+}^{N}$. Suppose first that $\tilde{u}$ is strictly positive in $\mathbb{R}_{+}^{N}$. By what we have already shown we know that $w_{\beta}^{(m)}\left(y, x_{N}\right)=w_{\beta}\left(y+y^{(m)}, x_{N}\right)>0$ in $\Sigma_{\beta}$ for all $\beta \leq \beta^{*}$. Hence the limit function $\tilde{w}^{\beta}=\lim _{m \rightarrow \infty} w_{\beta}^{(m)}$ is nonnegative in $\Sigma_{\beta}$ for all $\beta \leq \beta^{*}$.

So we can repeat the moving planes argument for $\tilde{u}$, and get $\tilde{\beta}^{*} \geq \beta^{*}$, where $\tilde{\beta}^{*}$ is to $\tilde{u}$ what $\beta^{*}$ is to $u$. Since $\tilde{w}^{\beta}$ satisfies an inequality like (3.15) we can apply the strong maximum principle and get, as before, that $\tilde{w}^{\beta}>0$ in $\Sigma_{\beta}$ for all $\beta \leq \tilde{\beta}^{*}$. On the other hand, by continuity and (3.16) we have $\tilde{w}^{\beta^{*}}\left(0, x_{N}^{0}\right)=0$, and $x_{N}^{0} \in\left(0, \beta^{*}-\varepsilon_{0}\right]$, a contradiction.

Suppose next $\tilde{u} \equiv 0$ in $\mathbb{R}_{+}^{N}$. We fix the rectangular domains

$$
\begin{aligned}
& Q_{1}=\left\{x \in \mathbb{R}_{+}^{N} \mid-1<x_{1}<1, \ldots .,-1<x_{N-1}<1, \varepsilon_{0}<x_{N}<2 \beta^{*}+1\right\}, \\
& Q_{2}=\left\{x \in \mathbb{R}_{+}^{N} \mid-2<x_{1}<2, \ldots .,-2<x_{N-1}<2, \frac{\varepsilon_{0}}{2}<x_{N}<2 \beta^{*}+2\right\},
\end{aligned}
$$

Since $u^{m}$ converges uniformly to zero in $Q_{2}$, we can suppose that $u^{(m)} \leq 1$ in $Q_{2}$.

We set

$$
\alpha_{m}=u^{(m)}\left(0, x_{N}^{(m)}\right) \quad \text { and } \quad v^{(m)}=\frac{u^{(m)}}{\alpha_{m}} .
$$

Now, by (3.13) the function $v^{(m)}$ satisfies

$$
\mathcal{M}_{\lambda, \Lambda}^{+}\left(D^{2} v^{(m)}\right)+\frac{f\left(u^{(m)}\right)}{u^{(m)}} v^{(m)}=0 \quad \text { in } Q_{2} .
$$

By applying Lemma 2.1 and Theorem 2.1 in these cubes we infer

$$
\sup _{Q_{1}} w^{(m)} \leq C_{1} \inf _{Q_{1}} w^{(m)} \leq C_{1}
$$


Next we recall that $w^{\beta^{*}} \geq 0$ in $\Sigma_{\beta^{*}}$, which implies

$$
v^{(m)}\left(y, x_{N}\right) \leq v^{(m)}\left(y, 2 \beta^{*}-x_{N}\right) \leq C_{1}, \quad \text { for }\left(y, x_{N}\right) \in \Sigma_{\beta^{*}}
$$

Hence

$$
\left\|v^{(m)}\right\|_{L^{\infty}(Q)} \leq C_{1}
$$

where

$$
Q=\left\{x \in \mathbb{R}_{+}^{N} \quad \mid \quad-1<x_{1}<1, \ldots .,-1<x_{N-1}<1,0<x_{N}<2 \beta^{*}+1\right\} .
$$

By applying Theorems 2.3 and 2.4 to $(3.17)$ we get that $v^{(m)} \rightrightarrows v$ on compacts and $v$ satisfies

$$
\mathcal{M}_{\lambda, \Lambda}^{+}\left(D^{2} v\right)+l v \leq 0
$$

where $l=\liminf _{t \searrow 0} \frac{f(t)}{t}$. By the strong maximum principle $v$ vanishes identically in $Q$ or $v>0$ in $Q$. The first possibility is excluded by $v\left(0, x_{N}^{0}\right)=1$.

Introduce the functions

$$
z^{\beta}\left(y, x_{N}\right)=v\left(y, 2 \beta-x_{N}\right)-v\left(y, x_{N}\right)
$$

defined in $\Sigma_{\beta} \cap \bar{Q}$ for all $\beta \leq \beta^{*}+1 / 2$. We have, by continuity,

$$
z^{\beta^{*}} \geq 0 \quad \text { and } \quad z^{\beta^{*}}\left(0, x_{N}^{0}\right)=0 .
$$

Since $\mathcal{M}_{\lambda, \Lambda}^{-}\left(D^{2} z^{\beta^{*}}\right)+l z^{\beta^{*}} \leq 0$ the strong maximum principle implies $z^{\beta^{*}}=0$ in $\Sigma_{\beta^{*}} \cap \bar{Q}$. This contradicts the fact that $v=0$ on $\left\{x_{N}=0\right\}$ and $v>0$ on $\left\{x_{N}=2 \beta^{*}\right\}$.

Theorem 3.2 Under the hypotheses of Theorem 3.1, if the problem

$$
\mathcal{M}_{\lambda, \Lambda}^{+}\left(D^{2} u\right)+f(u)=0
$$

has a nontrivial nonnegative bounded solution in $\mathbb{R}_{+}^{N}$ such that $u=0$ on $\partial \mathbb{R}_{+}^{N}$, then the same problem has a positive solution in $\mathbb{R}^{N-1}$.

Proof. Note that it is known how to deduce Theorem 3.2 from Theorem 3.1 when the Pucci operator is replaced by the Laplacian - then one multiplies by cut-off functions whose supports are strips going to infinity, and uses integration by parts. We can of course not use this approach, since Pucci's operators are not variational. 
Suppose $u$ is a solution of (3.18), $u \neq \equiv 0,0 \leq u \leq M$. For each $x=\left(y, x_{N}\right)$ in the strip $\Sigma_{1}=\left\{0<x_{N}<1\right\}$ we set

$$
u_{n}\left(y, x_{N}\right)=u\left(y, x_{N}+n\right) .
$$

Now $u_{n}$ satisfies the same equation as $u$ so, using once more the regularity and convergence results (Theorems 2.3 and 2.4), we see that $u_{n}$ converges uniformly on compact subsets of $\Sigma_{1}$ to a function $\widetilde{u}$ which satisfies

$$
\mathcal{M}_{\lambda, \Lambda}^{+}\left(D^{2} \widetilde{u}\right)+f(\widetilde{u})=0 \quad \text { in } \quad \Sigma_{1}
$$

However, the monotonicity result of Theorem 3.1 trivially implies that $\widetilde{u}$ is independent of the $x_{N}$-variable. This means that the last line and column of $D^{2} \widetilde{u}$ contain only zeros, so the $N$-dimensional Pucci operator applied to this matrix is actually $(N-1)$-dimensional, and we have (3.19) in $\mathbb{R}^{N-1}$.

\section{Existence results}

\subsection{The setting}

The proofs of our existence theorems are an application of degree theory for compact operators in cones. This theory, essentially developed by Krasnoselskii [23], has often been used to show that such operators possess fixed points. We are going to use an extension of Krasnoselskii results, due to Benjamin [3] and Nussbaum [28], in the form that have been stated in the paper of de Figueiredo, Lions and Nussbaum [19].

We start by recalling the abstract setting in [19]. Let $K$ be a closed cone with non-empty interior in the Banach space $(E,\|\cdot\|)$. Let $\Phi: K \rightarrow K$ and $F: K \times[0, \infty) \rightarrow K$ be compact operators such that $\Phi(0)=0$ and $F(x, 0)=$ $\Phi(x)$ for all $x \in K$. Then the following theorem holds (see Proposition 2.1 and Remark 2.1 in [19]).

Theorem 4.1 Assume there exist numbers $R_{1}>0, R_{2}>0$ and $T>0$ such that $R_{1} \neq R_{2}$, and

(i) $x \neq \beta \Phi(x)$ for all $0 \leq \beta \leq 1$ and $\|x\|=R_{1}$,

(ii) $F(x, t) \neq x$ for all $\|x\|=R_{2}$ and all $t \in[0,+\infty)$,

(iii) $F(x, t) \neq x$ for all $x \in \overline{B_{R_{2}}}$ and all $t \geq T$.

Then $\Phi$ has a fixed point $x \in K$ such that $\|x\|$ is between $R_{1}$ and $R_{2}$.

Note that (i) implies that $i_{C}\left(\Phi, B_{R_{1}}\right)=1$, while (ii) and (iii) imply $i_{C}\left(\Phi, B_{R_{2}}\right)=0$, where $i_{C}$ is the Krasnoselskii index and $B_{R}=\{x \in K$ : $\|x\|=R\}$, so Theorem 4.1 follows from the excision property of the index. 
We set $E=\{u \in C(\bar{\Omega}) \mid u=0$ on $\partial \Omega\}$ and $K=\{u \in E \mid u \geq 0$ in $\Omega\}$. It is clear that solving (1.2) is equivalent to finding a fixed point in $\mathrm{K}$ of $\Phi: K \rightarrow K$, defined by

$$
\Phi(u)(x) \stackrel{\text { def }}{=} \mathcal{L}(f(x, u(x))+\gamma u(x)), \quad x \in \Omega,
$$

where $\mathcal{L}$ is the inverse of $-\mathcal{M}_{\lambda, \Lambda}^{ \pm}\left(D^{2} \cdot\right)+\gamma$. It is easy to see, with the help of standard existence results for proper $(\gamma \geq 0)$ fully nonlinear elliptic equations, combined with Theorems 2.3 and 2.4, that $\mathcal{L}$ is well defined and compact (for details see [31]).

\subsection{Sublinear Equations. Proof of Theorem 1.1}

We define the operator $F$ as follows

$$
F(u, t)(x)=\mathcal{L}\left(f(x, u(x))+\gamma u(x)+t \varphi_{1}^{+}(x)\right),
$$

where $\varphi_{1}^{+}$is the positive eigenfunction of $\mathcal{M}_{\lambda, \Lambda}^{+}$, see Theorem 2.5.

Note that hypothesis $\left(H_{0}\right)$ implies that there exist constants $\varepsilon>0, r>0$ and $k>0$ such that for all $x \in \bar{\Omega}$

$$
f(x, t) \geq\left(\mu_{1}^{+}+\varepsilon\right) t \quad \text { if } t \leq r, \quad f(x, t) \leq\left(\mu_{1}^{+}-\varepsilon\right) t+k \quad \text { for all } t \geq 0 .
$$

We are going to show that conditions (i), (ii), (iii) in Theorem 4.1 are satisfied by $F(u, t)$, under the hypotheses of Theorem 1.1.

Let us prove (ii) and (iii). By the definition of $F$ we have

$$
F(u, t)=u \quad \Leftrightarrow \quad-\mathcal{M}_{\lambda, \Lambda}^{+}\left(D^{2} u\right)=f(x, u)+t \varphi_{1}^{+} .
$$

Hence, if $\|u\| \leq r$ we have

$$
\begin{aligned}
-\mathcal{M}_{\lambda, \Lambda}^{+}\left(D^{2} u\right) & \geq\left(\mu_{1}^{+}+\varepsilon\right) u+t \varphi_{1}^{+} \\
& \geq\left(\mu_{1}^{+}+\varepsilon\right) u \geq 0
\end{aligned}
$$

By the strong maximum principle $u \equiv 0$ or $u>0$ in $\Omega$. If $u>0(4.22)$ contradicts the definition of $\mu_{1}^{+}$(see Theorem 2.5). If $u \equiv 0,(4.21)$ implies $t=0$, so (ii) and (iii) are satisfied.

Next, we are going to prove (i). We claim that there exists $R>0$ such that for all $\beta \in[0,1]$

$$
u=\beta \Phi(u) \quad \text { implies } \quad\|u\| \leq R .
$$


Suppose this claim is false, that is, there exist sequences $\beta_{n} \in[0,1]$ (say $\left.\beta_{n} \rightarrow \beta\right)$ and $u_{n} \in K$ such that

$$
\left\|u_{n}\right\| \rightarrow \infty \quad \text { and } \quad-\mathcal{M}_{\lambda, \Lambda}^{+}\left(D^{2} u_{n}\right)+\gamma u_{n}=\beta_{n}\left(f\left(x, u_{n}\right)+\gamma u_{n}\right) .
$$

Set $v_{n}=\left\|u_{n}\right\|^{-1} u_{n}$. Then $v_{n}$ satisfies

$$
-\mathcal{M}_{\lambda, \Lambda}^{+}\left(D^{2} v_{n}\right)=g_{n}(x),
$$

where

$$
g_{n}=\beta_{n} \frac{f\left(x, u_{n}\right)}{u_{n}} v_{n}+\gamma\left(\beta_{n}-1\right) v_{n},
$$

so

$$
\left|g_{n}(x)\right| \leq\left(\beta_{n}\left(\mu_{1}^{+}-\varepsilon\right)+\gamma\left(\beta_{n}-1\right)\right) v_{n}+\frac{k \beta_{n}}{\left\|u_{n}\right\|},
$$

so $g_{n}$ is bounded. By imbedding theorems and Theorem 2.3 the sequence $v_{n}$ converges uniformly to a function $v$ such that $\|v\|=1$. By applying Theorem 2.4 to

$$
-\mathcal{M}_{\lambda, \Lambda}^{+}\left(D^{2} v_{n}\right) \leq\left(\beta_{n}\left(\mu_{1}^{+}-\varepsilon\right)+\gamma\left(\beta_{n}-1\right)\right) v_{n}+\frac{k \beta_{n}}{\left\|u_{n}\right\|}
$$

we get

$$
-\mathcal{M}_{\lambda, \Lambda}^{+}\left(D^{2} v\right) \leq\left(\mu_{1}^{+}-\varepsilon\right) v
$$

(since $\beta \in[0,1]$ ), which contradicts the maximum principle, Theorem 2.6.

In the end, we have proved (i), (ii) and (iii) with $R_{1}=R, R_{2}=r$.

\subsection{Superlinear Equations. Proof of Theorem 1.2 and Theorem 1.6}

Now we define the operator $F$ as

$$
F(u, t)(x)=\mathcal{L}(f(x, u(x)+t)+\gamma u(x)) .
$$

First we show that condition (i) in Theorem 4.1 is satisfied. This is the content of the following proposition.

Proposition 4.1 There is $R_{1}>0$ so that the equation

$$
\begin{array}{ll}
-\mathcal{M}_{\lambda, \Lambda}^{+}\left(D^{2} u\right)+\gamma u=\beta(f(x, u)+\gamma u) & \text { in } \Omega \\
u>0 \text { in } \Omega, \quad u=0 & \text { on } \partial \Omega,
\end{array}
$$

$\beta \in[0,1]$, has no solution $u$ with $0<\|u\|_{\infty}<R_{1}$. 
Proof. We argue by contradiction. Let $\left\{\left(u_{n}, \beta_{n}\right)\right\}_{n \in \mathbb{N}}$ be a sequence of positive solution to (4.23) such that $\left\|u_{n}\right\|_{\infty} \rightarrow 0$ as $n \rightarrow+\infty$. Define $v_{n}=$ $u_{n} /\left\|u_{n}\right\|_{\infty}$, then we have, as before, that $v_{n}$ satisfies

$$
-\mathcal{M}_{\lambda, \Lambda}^{+}\left(D^{2} v_{n}\right)=\beta_{n} \frac{f\left(u_{n}\right)}{u_{n}} v_{n}+\gamma\left(\beta_{n}-1\right) v_{n} \text { in } \Omega
$$

and $\left\|v_{n}\right\|_{\infty}=1$. By $\left(H^{0}\right)$ we can find $R_{1}>0$ such that

$$
f(u) \leq\left(\mu_{1}^{+}-\varepsilon\right) u \quad \text { if }\left\|u_{n}\right\|<R_{1} .
$$

Then we can argue as in the sublinear case, to conclude that we find $v_{n} \rightarrow v$ uniformly in $\bar{\Omega},\|v\|_{\infty}=1$, and $v$ satisfies $-\mathcal{M}_{\lambda, \Lambda}^{+}\left(D^{2} v\right) \leq\left(\mu_{1}^{+}-\varepsilon\right) v$, which contradicts the maximum principle.

In order to prove condition (iii) in Theorem 4.1 we state the following proposition.

Proposition 4.2 There exists a constant $T>0$ so that if

$$
F(u, t)=u \quad \Leftrightarrow \quad-\mathcal{M}_{\lambda, \Lambda}^{+}\left(D^{2} u\right)=f(x, u+t)
$$

possesses a solution $u \in K$, then

$$
0 \leq t \leq T
$$

Proof. By $\left(H^{0}\right)$ we can fix $\varepsilon>0$ and $T>0$ such that if $t \geq T$ then $f(t) \geq\left(\mu_{1}^{+}+\varepsilon\right) t$. Hence if (4.24) has a solution $u \in K$ for some $t \geq T$, then

$$
-\mathcal{M}_{\lambda, \Lambda}^{+}\left(D^{2}(u+t)\right) \geq\left(\mu_{1}^{+}+\varepsilon\right)(u+t) .
$$

By the definition of $\mu_{1}^{+}$this implies $u+t=0$, that is $t=0$ and $u \equiv 0$.

Note that the proof of this proposition also implies (ii) is verified for $t \geq T$. Then we complete the proof of Theorem 1.2 by noticing that condition (1.6) implies (ii) for $t \leq T$.

Finally, in order to prove Theorem 1.6 we are going to show that hypothesis $\left(f_{1}\right)$ implies (1.6).

Proposition 4.3 Let u be a $C^{2}(\Omega)$ solution of the equation (4.20) with $t \geq 0$. For each $t_{0}$ there exists a constant $C$ depending on $f$ and $\Omega$, such that if $u$ is a $C^{2}(\Omega)$ solution of the equation (4.24) with $0 \leq t \leq t_{0}$, then

$$
\|u\|_{\infty} \leq C
$$


Proof. We argue by contradiction. Let $\left\{\left(u_{n}, t_{n}\right)\right\}_{n \in \mathbb{N}}$ be a sequence of positive solution to (4.20) such that $0 \leq t_{n} \leq t_{0}$ (we can suppose $t_{n}$ converges), and $\left\|u_{n}\right\|_{\infty} \rightarrow+\infty$ as $n \rightarrow+\infty$.

Let us define

$$
v_{n}(x)=\frac{1}{M_{n}} u_{n}\left(x_{n}+x M_{n}^{\frac{1-p}{2}}\right),
$$

with $u_{n}\left(x_{n}\right)=M_{n}=\max _{\Omega} u_{n}$. Then $v_{n}$ satisfies

$$
-\mathcal{M}_{\lambda, \Lambda}^{+}\left(D^{2} v_{n}\right)=\frac{f\left(u_{n}+t_{n}\right)}{M_{n}^{p}} \quad \text { in } \quad \Omega_{n}=M_{n}^{\frac{p-1}{2}}\left(\Omega-x_{n}\right),
$$

and $\left\|v_{n}\right\|_{\infty}=1$. It is standard to see that $\Omega_{n}$ tends to $\mathbb{R}^{N}$ or $\mathbb{R}_{+}^{N}$. By the regularity result, Theorem 2.3, we have that, up to a subsequence, $v_{n} \rightrightarrows v$ in compact sets of $\mathbb{R}^{N}$ or $\mathbb{R}_{+}^{N}$. By hypothesis $\left(f_{1}\right)$ we have that $f\left(u_{n}+t_{n}\right) / M_{n}^{p} \rightarrow C^{*} v^{p}$, so $v \in K$ is classical bounded solution of

$$
\mathcal{M}_{\lambda, \Lambda}^{+}\left(D^{2} v\right)+C^{*} v^{p}=0 \text { in } \mathbb{R}^{N},
$$

or of

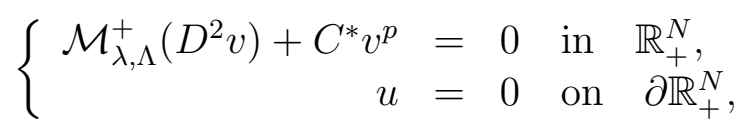

with $p \leq p^{+}$. However, this contradicts Theorem 1.3 or Theorem 1.5, since we have $\|v\|=1$.

\section{References}

[1] A.D. ALEXANDROV, A characteristic property of the spheres, Ann. Mat. Pura Appl. 58 (1962), 303-354.

[2] M. BARDI - F. DA LIO, On the strong maximum principle for fully nonlinear degenerate elliptic equations. Arch. Math. (Basel) 73 (1999), 276-285.

[3] T.B. BENJAMIN, A unified theory of conjugate flows, Phil. Trans. Royal Soc. 269A (1971), 587-643.

[4] A. BENSOUSSAN - J.L. LIONS, Applications of variational inequalities in stochastic control. Translated from French, In: "Studies in Mathematics and its Applications" 12, North-Holland Publishing Co., AmsterdamNew York, 1982. 
[5] H. BERESTYCKI - L. NIRENBERG, On the method of moving planes and the sliding method. Bull. Soc. Br. Mat. Nova Ser. 22 (1991), 1-37.

[6] L. CAFFARELLI - H. BERESTYCKI - L. NIRENBERG, Further qualitative properties for elliptic equations in unbounded domains. Dedicated to Ennio De Giorgi, Ann. Scuola Norm. Sup. Pisa Cl. Sci. 25(1-2) (1997), 69-94.

[7] J. BUSCA - M. ESTEBAN - A. QUAAS, Nonlinear eigenvalues and bifurcation problems for Pucci's operator, to appear in Ann Inst. Henri Poincaré, Analyse non linéaire.

[8] X. CABRE, On the Alexandrov-Bakelman-Pucci estimate and the reversed Hölder inequality for solutions of elliptic and parabolic equations, Comm. Pure and Appl. Math. 48 (1995), 539-570.

[9] X. CABRE, Topics in regularity and qualitative properties of solutions of nonlinear elliptic equations, Discr. Cont. Dyn. Syst. 8(2) (2002), 331359.

[10] X. CABRE - L. CAFFARELLI, Fully Nonlinear Elliptic Equation, American Mathematical Society, Colloquium Publication, Vol. 43, 1995.

[11] L. CAFFARELLI - M.G. CRANDALL - M.KOCAN - A. ŚWIECH, On viscosity solutions of fully nonlinear equations with measurable ingredients, Comm. Pure Appl. Math 49 (1996), 365-397.

[12] M. CRANDALL - H. ISHII - P.L. LIONS, User's guide to viscosity solutions of second order partial differential equations, Bull. AMS 27(1) (1992), 1-67.

[13] A. CUTRI - F. LEONI, On the Liouville property for fully nonlinear equations. Ann Inst. Henri Poicaré, Analyse non linéaire 17(2) (2000), $219-245$.

[14] E.N. DANCER, Some notes on the method of moving planes, Bull. Austral. Math. Soc. 46 (1992), 425-434.

[15] K. DEIMLING, Nonlinear functional analysis, Springer-Verlag, 1985.

[16] P. FELMER - A. QUAAS, Critical Exponents for the Pucci's Extremal Operators, C.R. Acad. Sci. Paris (I) 335 (2002), 909-914. 
[17] P. FELMER - A. QUAAS, On Critical exponents for the Pucci's extremal operators, Ann Inst. Henri Poincaré, Analyse non linéaire 20(5) (2003), 843-865.

[18] P. FELMER - A. QUAAS, Positive solutions to 'semilinear' equation involving the Pucci's operator, to appear in J. Diff Eq.

[19] D.G DE FIGUEIREDO - P.L. LIONS - R.D. NUSSBAUM, A priori estimates and existence of positive solutions of semilinear elliptic equation, J. Math. Pures Appl. 61 (1982), 41-63.

[20] B. GIDAS - W.M. NI - L. NIRENBERG, Symmetry and related properties via the maximum principle, Comm. Math. Phys. 6 (1981), 883-901.

[21] B. GIDAS - J. SPRUCK, Global and local behavior of positive solutions of nonlinear elliptic equations, Comm. Pure Appl. Math. 34 (1981), 525-598.

[22] D. GILBARG - N. S. TRUDINGER, Elliptic partial differential equation of second order, 2nd ed., Springer-Verlag, 1983.

[23] M.A. KRASNOSELSKII, Positive solutions of operator equations, P. Noordhoff, Groningen, 1964.

[24] N.V. KRYLOV, Nonlinear elliptic and parabolic equations of second order, Coll. Math. and its Appl., Reidel, 1987.

[25] P.L. LIONS, On the existence of positive solutions of semilinear elliptic equation, SIAM Review 24(4) (1982), 441-446.

[26] P.L. LIONS, Optimal control of diffusion processes and Hamilton-JacobiBellman equations. I. The dynamic programming principle and applications, Comm. Part. Diff. Eq. 8 (1983), 1101-1174.

[27] J. MAWHIN, Leray-Schauder degree : a half-century of extensions and applications, Topol. Meth. Nonl. Anal. 14(2) (1999), 195-228.

[28] R.D. NUSSBAUM, Periodic solutions of some nonlinear, autonomous functional differential equations, J. Diff. Eq. 14 (1973), 360-394.

[29] C. PUCCI, Operatori ellittici estremanti, Ann. Mat. Pure Appl. 72 (1966), 141-170.

[30] P. RABINOWITZ, Minimax methods in critical point theory with applications to differential equations, CMBS Series in Math. 65, 1986. 
[31] A. QUAAS, Existence of Positive Solutions to a 'semilinear' equation involving the Pucci's operator in a convex domain, Diff. Int. Eq. 17 (2004), 481-494.

[32] J. SERRIN, A symmetry theorem in potential theory, Arch. Rat. Mech. Anal. 43 (1971), 304-318.

\author{
Alexander QUAAS \\ Departamento de Matemática, Universidad Santa Maria \\ Avenida España 1680, Casilla 110-V, Valparaíso, Chile \\ e-mail : alexander.quaas@usm.cl \\ Boyan SIRAKOV \\ Université Paris 10, 92001 Nanterre Cedex, France \\ and CAMS, EHESS, 54 bd Raspail, 75006 Paris, France \\ e-mail : sirakov@ehess.fr
}

